\title{
Association between negative FB and mortality in SICU
}

\author{
Xuping Cheng ${ }^{1} \cdot$ Kailei $\mathrm{Du}^{1}$ (1)
}

Received: 19 June 2019 / Accepted: 2 November 2019 / Published online: 14 November 2019

(c) Japanese Society of Anesthesiologists 2019

To the Editor:

In a recent cohort study, Dr. Oh [1] reported that compared to normal FB (0-5\%), the mortality rate in the negative FB $(<0 \%)$ was comparable in SICU patients. Several limitations need to be addressed. First, translating a continuous variable (FB) into a category variable may weaken the statistical power to detect a true association. For instance, Balakumar [2] found that compared with even FB, both positive and negative FB were associated with higher mortality in critically ill patient. Whether there is a similar relationship in SICU patients cannot be inferred. Second, $0-5 \%$ of FB was defined as normal FB while the cut-off value was around $1 \%$ in Fig. 2 (panel B), and under this grouping method, the patients were unevenly distributed (5423 (68.7\%) in normal FB, 1616 (20.4\%) in negative FB). Simply categorizing these 5423 patients into one group may lose important information. Thus, we performed an additional analysis using data from MIMIC III database. Patients were divided into two subgroups according to the $48 \mathrm{~h}-\mathrm{FB}(1 \% \leq \mathrm{FB}<5 \%$ and $\mathrm{FB}<1 \%)$. Quartile method was used. In the subgroup with
$\mathrm{FB} \geq 1 \%$, the odds ratio (OR) of mortality stepwise increased from Q2 (OR 1.30, 95\% CI 0.92-1.82) to Q4 (OR 2.15, 95\% CI 1.57-2.93), referred to Q1, which indicated slightly increased FB was still a risk factor of mortality. In the subgroup with $48 \mathrm{~h}-\mathrm{FB}<1 \%$, achieving more negative $\mathrm{FB}$ was not associated with mortality (Table S1).

\section{References}

1. Oh TK, Song IA, Do SH, Jheon S, Lim C. Association of perioperative weight-based fluid balance with 30-day mortality and acute kidney injury among patients in the surgical intensive care unit. J Anesth. 2019;33(3):354-63.

2. Balakumar V, Murugan R, Sileanu FE, Palevsky P, Clermont G, Kellum JA. Both positive and negative fluid balance may be associated with reduced long-term survival in the critically ill. Crit Care Med. 2017;45(8):e749-e757757.

Publisher's Note Springer Nature remains neutral with regard to jurisdictional claims in published maps and institutional affiliations.

This comment refers to the article available online at https://doi. org/10.1007/s00540-019-02630-8.

Electronic supplementary material The online version of this article (https://doi.org/10.1007/s00540-019-02709-2) contains supplementary material, which is available to authorized users.

Kailei Du

dkldyrm@163.com

1 Department of Intensive Care, Dongyang People Hospital, No. 60, Wuning Road, Jinhua, Zhejiang, China 